\title{
Comparative Study of AODV andDSR Routing Protocols forGateway Placement Optimization
}

\author{
Jamal Muafaq Hameed Al-Bayati \\ PhD Student of Computer Science, Faculty of Mathematics, Belgrade University \\ jamrafab@yahoo.com
}

\begin{abstract}
This paper considers mobile ad-hoc network (MANET) in which mobile nodes can reach the Internet via stationary gateway node. The gateway works as bridge between MANET and the Internet. Several studies show that location of the gateway inside network topology has a significant effect on overall network performance. Two different scenarios, with varying number of nodes and two gateway locations, have been considered under the assumption that all mobile nodes are connected to Internet through the same gateway. Simulation has been done using NS-2 software, producing a computer model of AODV and DSR routing protocols. Comparison of these routing protocols is performed in terms of Average (end-to-end) delay, Throughput, Normalized routing load and Packet delivery ratio metrics. Simulation results suggest that AODV routing protocol has better performance in both scenarios. In addition, the results suggest that location of gateway at the center gives better results compared to gateway positioned at the edge.
\end{abstract}

\section{Index Terms}

Networks, MANET, Gateway Placement, AODV, DSR, NS2.

\section{Introduction}

Mobile ad hoc networks (MANETs) comprise autonomous mobile nodes that can communicate to each other over wireless link and with the ability to move and even to switch off arbitrarily. These networks can be set in isolation or can be connected to other networks. ${ }^{1}$ The interconnection of internet and MANET is provided by a gateway node. ${ }^{2}$

Gateway placement has an important effect on network performance. It represents an important part of wireless network design. Placing more than one gateway, leads to increment the throughput and reduces overcrowding, but on the other hand it leads to also increment the interference and cost.

This paper shows characteristics of ad-hoc routing protocols, DSR and AODV, related to the gateway placement. Performance of these two routing protocols has been evaluated based on throughput, packet delivery ratio, average endto-end delay and normalized routing load metrics by using NS-2 simulator in UNIX environment.

\section{Related work:}

Several routing protocols have been analyzed comparatively for their performance behavior in different settings and scenarios. Mina VajedKhiavi, ShahramJamali andSajjadJahanbakhshGudakahrizevaluated various routing protocols by extensive simulations in Ns-2 simulator as well as the impact of pause time and number of nodes on performance. ${ }^{3}$ In this study, performance was measured in terms of Packet Delivery Ratio, Network Life Time, System Life Time, End-to-end Delay and Routing Overhead.

The main object of that research was effect of mobility models on the performance of three MANET on-demand reactive routing protocols: AODV, DYMO and DSR. ${ }^{4}$ Mobility models used in this work are: Random Waypoint mobility model and Group mobility model. Performance evaluations are analyzed by applying varying network size, differing pause time and diverse velocity. Performance analysis has been done based on different network metrics, such as packet delivery ratio, throughput, average end-to-end delay and average jitter.

The response of multiple routing protocols to the placement of gateway in the Wireless Mesh topologies as a function of routing overhead, network latency and Packet Delivery Ratio are also tested. ${ }^{5}$

\footnotetext{
${ }^{1}$ Miklos Molnar and Raymond Marie, Stability Oriented Routing in Mobile Ad-Hoc Networks Based on Simple Automatons, Intech, 2011.

2 Antonio J. Yuste, Alicia Triviño, Eduardo Casilari, Francisco D. Trujillo, An Optimized MANET Gateway Discovery Based on Fuzzy Logic, Springer, Volume 84, pp 273-282, 2010.

${ }^{3}$ Mina Vajed Khiavi, Shahram Jamali, Sajjad Jahanbakhsh Gudakahriz, Performance Comparison of AODV, DSDV, DSR and TORA Routing Protocols in MANETs, International Research Journal of Applied and Basic Sciences, Vol. 3 (7), 1429-1436, 2012.

${ }^{4}$ Shruti Bajaj, Er. Rajdeep Singh, Er. Parveen Kakkar, Investigation of Mobility Model Against Reactive Routing Protocols in MANETs, International Journal of Computer \& Technology Vol. 8, No. 1, June $20^{\text {th }}, 2013$.

${ }^{5}$ Harpreet Sandhu, Roopali Garg, Performance Evaluation of Gateway Discovery Routing Protocols in MANETs, International Journal of Computer Science, Engineering and Applications (IJCSEA) Vol. 2, No. 3, June 2012.
} 
Two different scenarios have been simulated with various numbers of network flows and performance of these routing protocols has been analyzed. Simulation results suggest that proactive routing protocol outperforms the reactive one, while gateways placed at the center of the network topology enhance network performance.

AODV, DSDV and I-DSDV with NS-2 package are simulated and compared by packet delivery ratio, end-to-end delay and routing overhead metrics in different environments with varying number of nodes, speed time and pause time. ${ }^{6}$ Simulation results show that I-DSDV, compared to DSDV, reduces the number of dropped data packets, but increases overhead at higher rates of node mobility. Although, it still can't compete with AODV in higher node speed and number of node.

Routing protocols for mobile ad-hoc networks - Destination Sequenced Distance Vector (DSDV), table driven protocol and Ad-hoc On-Demand Distance Vector routing (AODV) are introduced, too. ${ }^{7}$ Performance of these protocols has been evaluated based on packet delivery ratio and average delay with varying number of sources and pause time. Simulation results show that AODV routing protocol is among good choices for communication.

\section{Routing Protocols in MANETs}

Two routing protocols, DSR and AODV,were topics of this paper. Brief summaries of these protocols are provided below.

a) Dynamic Source Routing Protocol (DSR)

DSR is an efficient and simple routing Protocol designed precisely for use in multi hop wireless Ad hoc Networks of mobile nodes. In DSR, the network is fully self-organizing and self-configuring, requiring no existing network infrastructure or administration. DSR Protocol lets nodes to dynamically discover a source route across multiple network hops to any destination in the Ad-hoc network. Each packet sent then carries in its header complete, ordered list of nodes through which the packet must pass, allowing packet routing to be trivially loopfree and no need for up-to-date routing information in the intermediate nodes through which the packet is forwarded. ${ }^{8}$

b) Ad-hoc On Demand Distance Vector (AODV)

AODV is a distance vector type routing where it does not comprise nodes to maintain routes to destination that are not on active path. AODV uses different route messages like Route Request, Route Replies and Route Errors to discover and maintain links. It also uses a destination sequence number for every route created by destination node for any request to the nodes. The route with maximum sequence number is chosen. To select a new route, the source node broadcasts a Route Request message to the network till destination is reached or a node with fresh route is found. Then Route Reply is sent back to the source node. ${ }^{9}$

\section{Performance Metrics}

We considered multiple metrics in analyzing performance of routing protocols. These metrics represent mobility model and communicating traffic pattern.

- Throughput:the total number of received data packets divided by the total duration of simulation time. ${ }^{10}$

- Normalized Routing Load:the total number of routing packets divided by the total number of received packets. $^{11}$

- Average End-to-end Delay: The average time taken by the difference of packet sent and received time by the total time difference of the total number of packet received. ${ }^{12}$

\footnotetext{
${ }^{6}$ Abdul Hadi Abd Rahman and Zuriati Ahmad Zukarnain, Performance Comparison of AODV, DSDV and I-DSDV Routing Protocols in Mobile Ad Hoc Networks, European Journal of Scientific Research, Vol. 31, No. 4, pp. 566-576, 2009.

${ }^{7}$ R. Balakrishna, S. Jeyabalan, Dr U. Rajeswar Rao, Dr. T. K. Basak, and Dr V.CYRILRAJ, Performance Issues on $A O D V$ and DSDV for MANETS, Journal of Theoretical and Applied Information Technology (JATIT), Vol. 17, No. 2, 2010.

${ }^{8}$ Surendra H. Raut, Hemant P. Ambulgekar, Proactive and Reactive Routing Protocols in Multihop Mobile Ad hoc Network, International Journal of Advanced Research in Computer Science and Software Engineering, Volume 3, Issue 4, April 2013.

9 Amit Shrivastava, Aravinth Raj, Shanmogavel, Avinash Mistry, Nitin Chander Prashanth Patlolla, Vivek Yadlapalli, Overview of Routing Protocols in MANET's and Enhancements in Reactive Protocols, Department of Computer Science, Lamar University.

${ }^{10}$ Ahmed Al-Maashri, Mohamed Ould-Khaoua, "Performance analysis of MANET routing protocols in the presence of self-similar traffic," IEEE Conference on Local Computer Networks, 2006.

${ }^{11}$ Ibid.
} 
- Packet Delivery Ratio is defined as the ratio of the number of received data packet to the destination. This explains the level of data dispatched to the destination.

\section{Simulations and Results}

Our simulations have been performed in two scenarios by changing locations of Gateway. Network Simulator NS2 has been used to simulate the network in LINUX environment.

\section{The First Scenario:}

Single gateway are located in the center of network with different numbers of nodes $(50,100$ and 150) are displayed in the network. All these nodes are sent and received packets to the same destination (gateway) by using different twoRouting Protocols (AODV and DSR). Table1illustrates parameters used in the above mentioned scenarios.

Table 1: Simulation parameters

\begin{tabular}{|c|c|}
\hline PARAMETER & VALUE \\
\hline Routing Protocols & AODV,DSR \\
\hline Number of Nodes & $50,100,150$ \\
\hline Radio model & Two-ray Ground \\
\hline Node Placement & Random node placement \\
\hline Gateway Placement & Center \\
\hline Simulation Time (Sec) & 60.0 \\
\hline Packet Size & $4 \mathrm{~K}$ \\
\hline Simulation Area size & $2000 \mathrm{~m} \times 2000 \mathrm{~m}$ \\
\hline Simulator & Ns-2.35 \\
\hline Performance Metrics & $\begin{array}{c}\text { Packet Delivery Ratio, End-to-end delay, Throughput, } \\
\text { Normalized Routing Load. }\end{array}$ \\
\hline Maximum packet in ifq & 50 \\
\hline Channel Type & Wireless channel \\
\hline Application Traffic & FTP \\
\hline MAC & Mac/802_11 \\
\hline
\end{tabular}

Performance of these two routing protocols is analyzed based on throughput, average end-to-end delay, normalized routing load and packet delivery ratio, as shown in figures (1-4).These results, in general, clearly show that AODV has a better performance rather than DSR in case: average end-to-end delay, packet delivery ratio. The performance of DSR was better rather than AODV in case: throughput and normalized routing load when the number of nodes less than 150.

${ }^{12}$ D. Loganathan, P. Ramamoorthy, Performance Analysis of Enhanced DSDV Protocol for Efficient Routing In Wireless Ad Hoc Networks, Research Inventy: International Journal Of Engineering And Science Vol. 2, Pp 01-08, April 2013. 


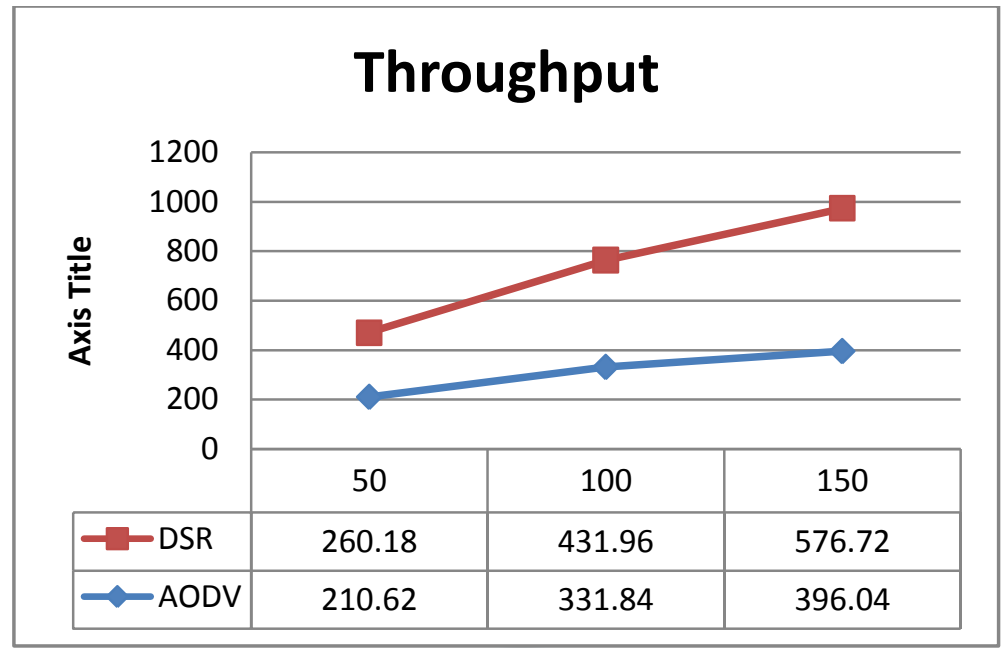

Figure 1: Throughput Vs. No. of nodes in scenario 1

\section{Average End-To-End Delay}

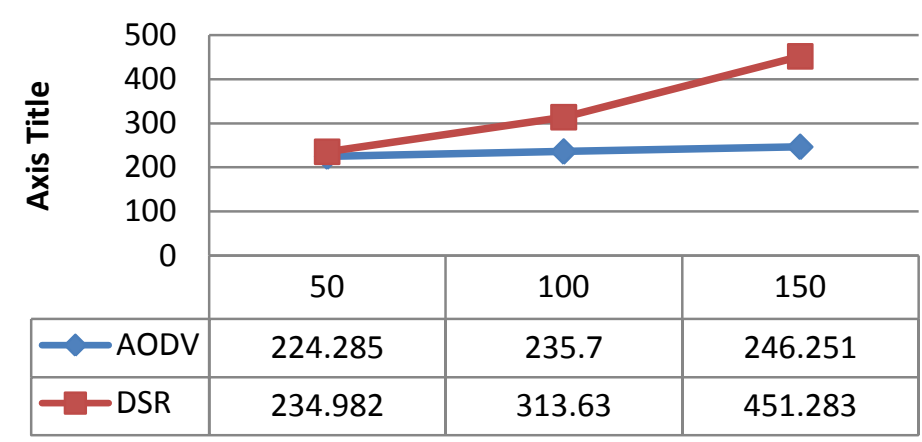

Figure 2: Average end-to-end delay Vs. No. of nodes in scenario 1

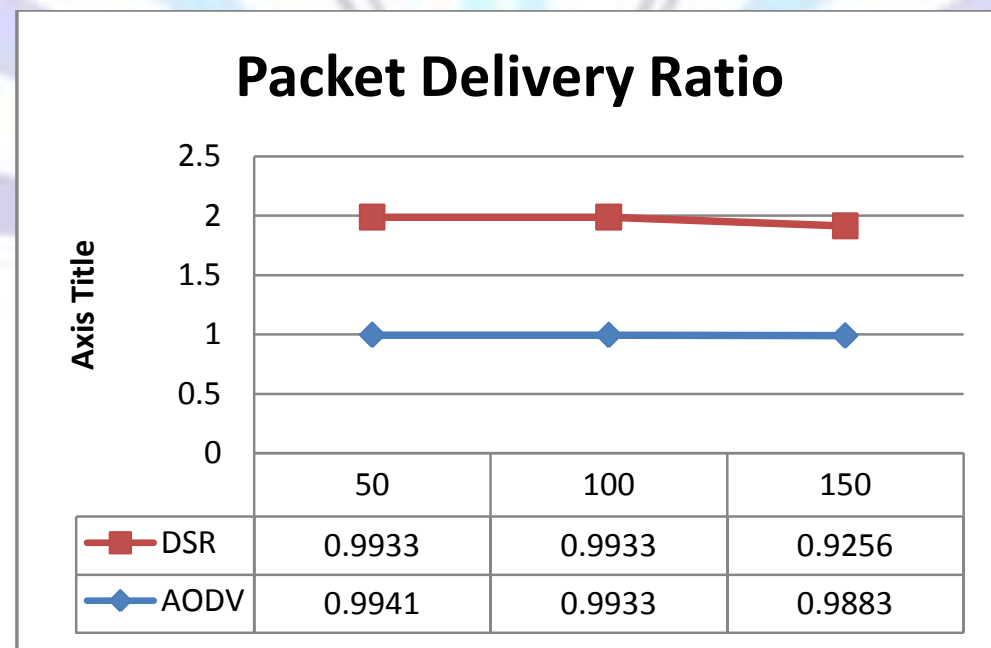

Figure 3: Packet Delivery Ratio Vs. No. of nodes in scenario 1 


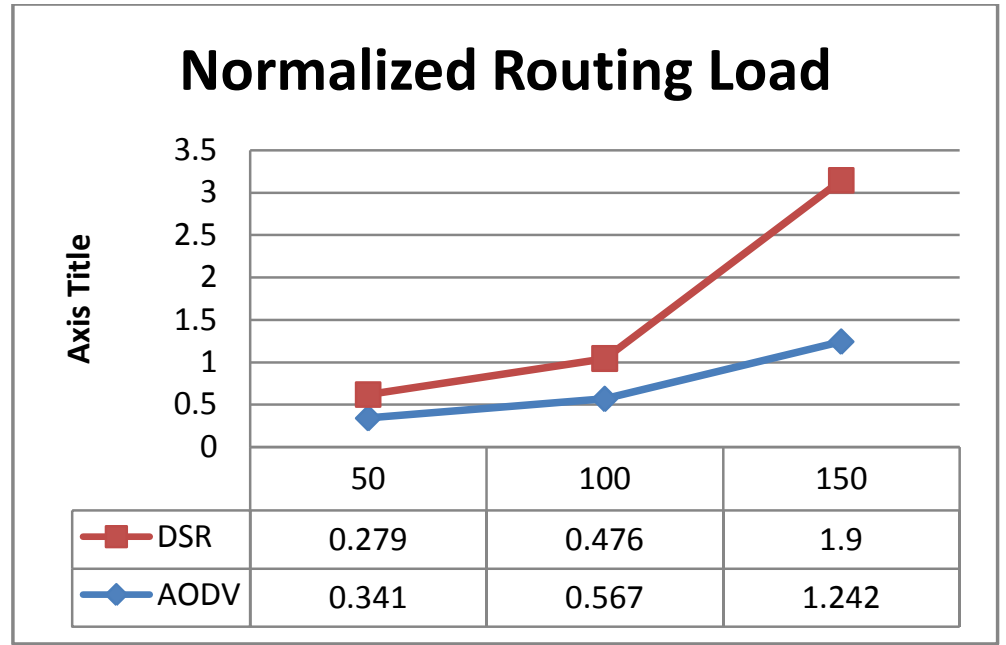

Figure 4: Normalized routing load Vs. No. of nodes in scenario 1

\section{The Second Scenario:}

In this scenario, different numbers of nodes $(50,100$ and 150) were also considered. Furthermore, we studied high traffic, the destination (gateway) located at the edge of network and we made all the nodes send and receive packets to the same destination.

Table 2 illustrates the parameters used in the second scenario.

\begin{tabular}{|c|c|}
\hline PARAMETER & VALUE \\
\hline Routing Protocols & AODV,DSR \\
\hline Number of Nodes & $50,100,150$ \\
\hline Radio model & Two-ray Ground \\
\hline Node Placement & Random node placement \\
\hline Gateway Placement & Edge \\
\hline Simulation Time (Sec) & 60.0 \\
\hline Packet Size & $4 \mathrm{~K}$ \\
\hline Simulation Area size & $2000 m \times 2000 m$ \\
\hline Performance Metrics & $\begin{array}{l}\text { Packet Delivery Ratio, End to End delay, } \\
\text { Throughput, Normalized Routing Load. }\end{array}$ \\
\hline Maximum packet in ifq & 50 \\
\hline Channel Type & Wireless channel \\
\hline Application Traffic & FTP \\
\hline MAC & Mac/802_11 \\
\hline
\end{tabular}

Performance of these two routing protocols is also analyzed based on throughput, average end-toend delay, normalized routing load and packet delivery ratio, as shown in figures (5-8). These results highlightthat AODV has better performance than DSR in average end-to-end Delay, Packet Delivery Ratio, Normalized Routing Load and throughput, in overall.Additionally, the DSR fails in increasing number of nodes. 


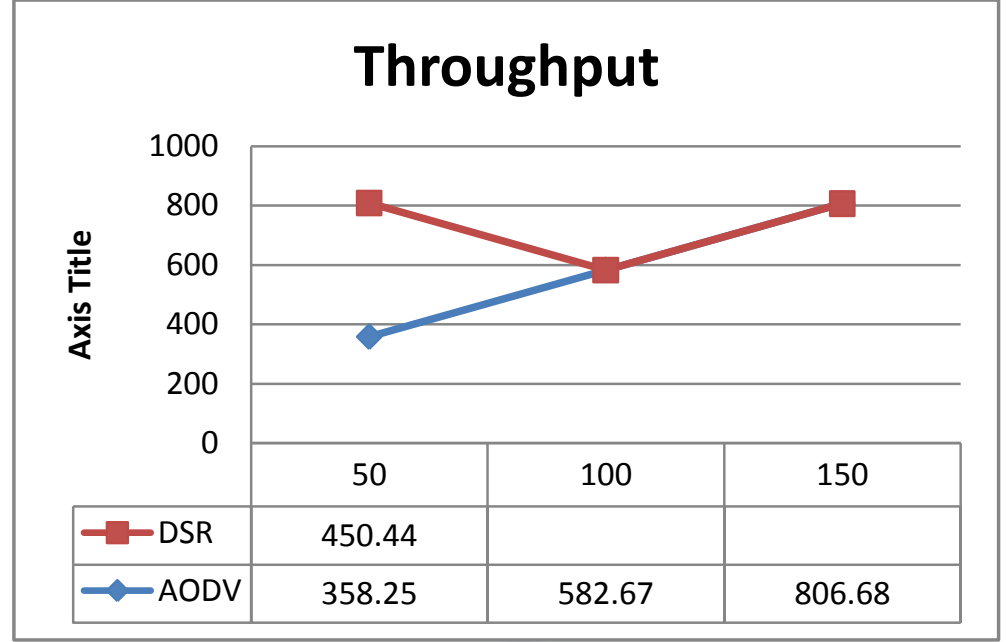

Figure 5: Throughput Vs. No. of nodes in scenario 2

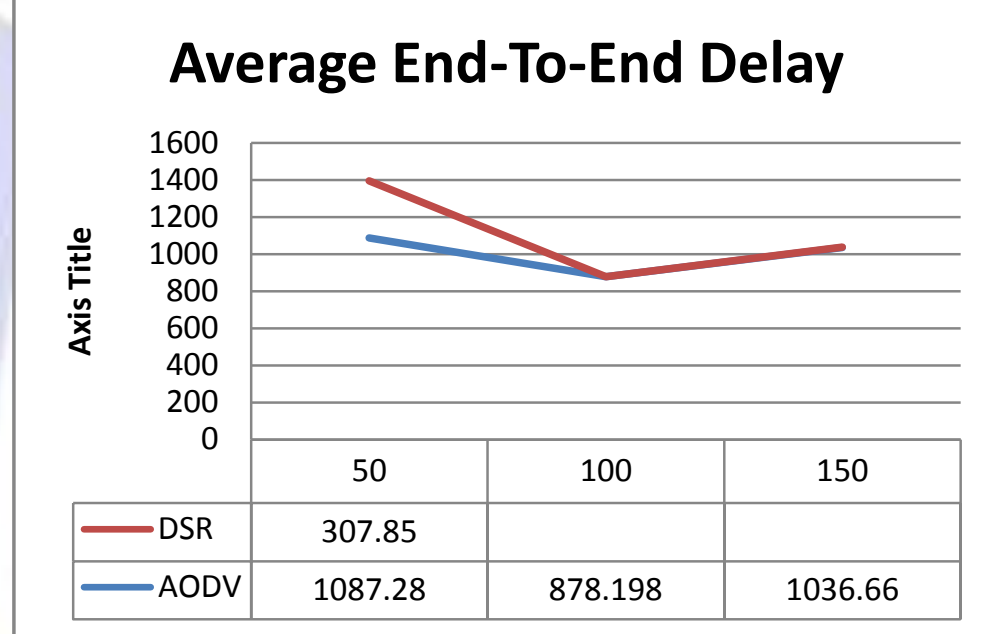

Figure 6: Average end-to-end delay Vs. No. of nodes in scenario 2

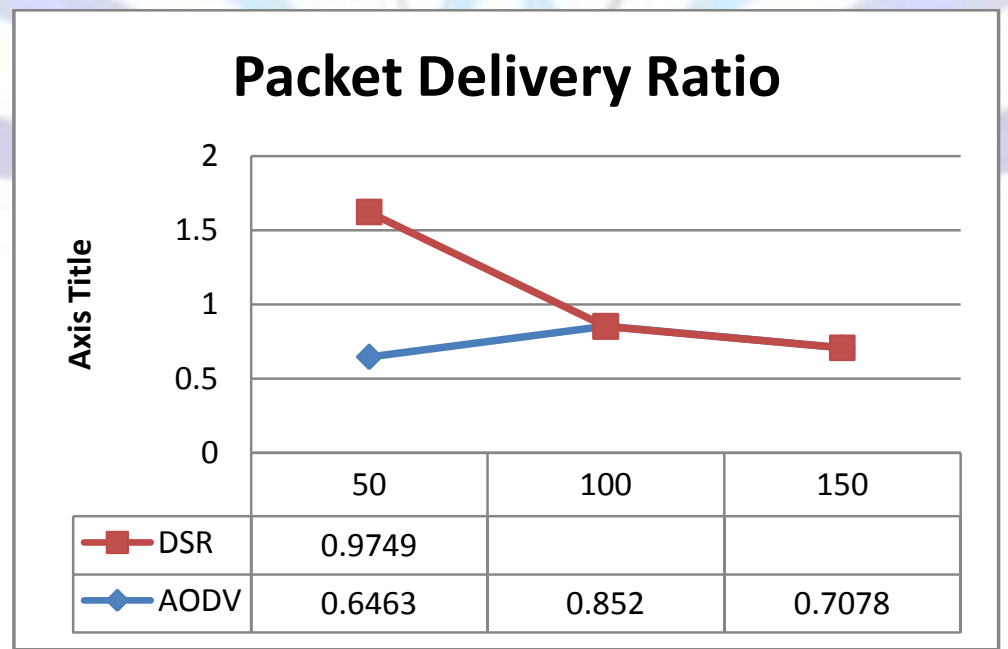

Figure 7: Packet Delivery Ratio Vs. No. of nodes in scenario 2 


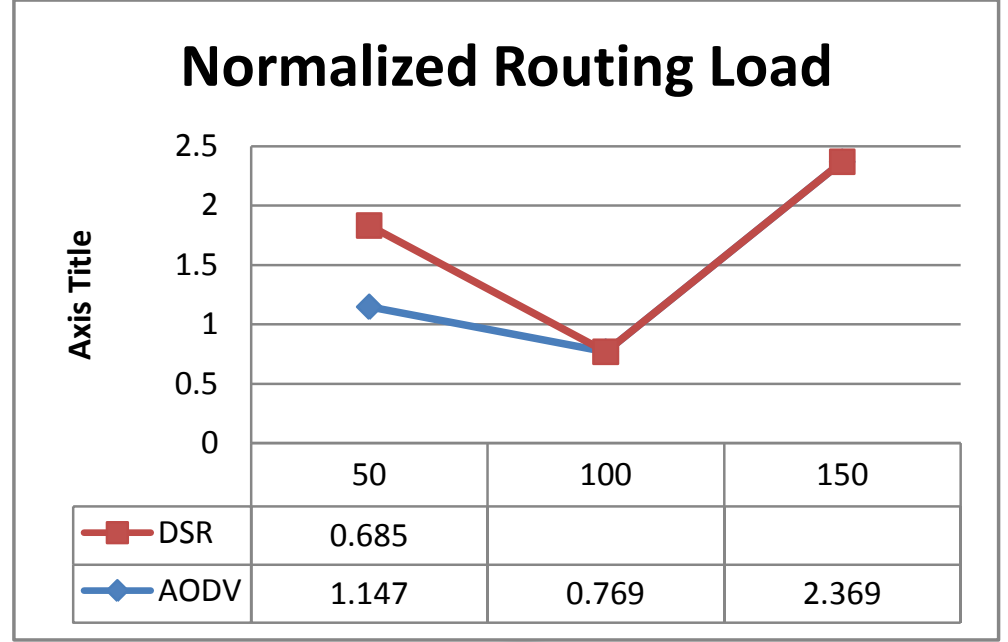

Figure 8: Normalized routing load Vs. No. of nodes in scenario 2

\section{CONCLUSION}

According to achieved experimental results, we concluded that AODV has a better performance than DSR in two scenarios. It is also enclosed that the gateway location in the center gives better results than the location in the edge. Furthermore, presented results prove that DSR fails with increasing number of nodes and AODV has lower average end-to-end delay. 